\title{
Intestinal heat shock protein 110 regulates expression of CD1d on intestinal epithelial cells
}

\author{
Sean P. Colgan, ${ }^{1}$ Richard S. Pitman, ${ }^{2}$ Takashi Nagaishi, ${ }^{2}$ Atsushi Mizoguchi, ${ }^{3}$ \\ Emiko Mizoguchi, ${ }^{3}$ Lloyd F. Mayer, ${ }^{4}$ Ling Shao, ${ }^{4}$ R. Balfour Sartor, ${ }^{5}$ \\ John R. Subjeck, ${ }^{6}$ and Richard S. Blumberg ${ }^{2}$ \\ ${ }^{1}$ Center for Experimental Therapeutics, and \\ ${ }^{2}$ Division of Gastroenterology, Brigham and Women's Hospital and Harvard Medical School, Boston, Massachusetts, USA \\ ${ }^{3}$ Immunopathology Unit, Massachusetts General Hospital and Harvard Medical School, Boston, Massachusetts, USA \\ ${ }^{4}$ Immunobiology Center, Mount Sinai School of Medicine, New York, New York, USA \\ ${ }^{5}$ University of North Carolina, Chapel Hill, North Carolina, USA \\ ${ }^{6}$ Department of Molecular and Cellular Biophysics, Roswell Park Cancer Institute, Buffalo, New York, USA
}

\begin{abstract}
CD1d is expressed on the surface of professional and nonprofessional APCs, including intestinal epithelial cells (IECs), for a role in the presentation of glycolipid-based antigens to subsets of T cells. The mechanisms that regulate CD1d expression in any cell type are unknown. To investigate the possibility that expression of CD1d is influenced by exogenous factors present within the intestinal lumen, CD1d expression was analyzed in several IEC lines after culturing in the presence of lumenal contents (LC) of the normal human intestine. Exposure of the colon-derived cell lines T84, HT-29, and Caco-2 to soluble LC resulted in a marked induction of CD1d expression as determined by RT-PCR, confocal microscopy, cell surface ELISA, and Western blot analysis. Similarly, exposure of human IECs to LC isolated from mice bred in both specific pathogen-free and germfree conditions also resulted in the induction of CD1d expression, with the maximum CD1d-inducing activity observed in the small intestine. Biochemical and biophysical characterization of the human CD1dinducing activity identified heat shock protein 110 (Hsp110) as a major functional component of the LC that contributes to CD1d surface regulation, and immunolocalization studies revealed Hsp110 expression in subsets of human IECs in vivo. These data support the presence of a novel autocrine pathway of CD1d regulation by Hsp110.
\end{abstract}

J. Clin. Invest. 112:745-754 (2003). doi:10.1172/JCI200317241.

\section{Introduction}

CD1d belongs to the CD1 family of proteins that share structural and functional homology to the MHC class I and class II molecules $(1,2)$. CD1d is expressed by a majority of thymocytes, resting B cells, DCs, and monocytes $(1,3)$. In addition, CD1d expression is observed in a variety of epithelial cell types, including hepatocytes and intestinal epithelial cells (IECs) $(4,5)$. Although its role has not been clearly defined in epithelia, CD1d is known to associate with glycolipid antigens and present them to subsets of CD1d-restricted $\mathrm{T}$ cells bearing NK cell markers (NK-T cells) (2). Activation of CD1d-

Received for publication October 24, 2002, and accepted in revised form June 3, 2003.

Address correspondence to: Richard S. Blumberg, Division of Gastroenterology, Brigham and Women's Hospital, Thorn Building, Room 1410, 75 Francis Street, Boston, Massachusetts 02115, USA. Phone: (617) 732-6912; Fax: (617) 264-5185;

E-mail: rblumberg@partners.org.

Sean P. Colgan and Richard S. Pitman contributed equally to this work.

Conflict of interest: The authors have declared that no conflict of interest exists.

Nonstandard abbreviations used: intestinal epithelial cell (IEC); heat shock protein 110 (Hsp110); lumenal contents (LC); specific pathogen-free (SPF); Toll-like receptor (TLR). restricted cells in vivo by association of CD1d with a model lipid antigen, $\alpha$-galactosylceramide, regulates colitis in a chemically induced disease model $(6,7)$. In these studies on colitis, $\alpha$-galactosylceramide was observed to localize to the surface of the intestinal epithelium, raising the possibility that $\mathrm{CD} 1 \mathrm{~d}$ was functional in this site in vivo. Moreover, intestinal epithelial CD1d can signal the production of IL-10, which, through autocrine pathways, can protect epithelia from the deleterious influence of cytokines such as IFN- $\gamma(8)$. Such data have implicated CD1d as a molecule capable of facilitating mechanisms that regulate mucosal inflammation and highlight the CD1d-bearing IEC as a candidate cell type in this immune regulation.

Immunostaining and biochemical analysis of tissues from the human small and large intestine have revealed the presence of CD1d on both the apical and the basolateral membranes of IECs (9). In addition, CD1d is observed intracellularly within subapical regions in these cells. Relative to the levels of CD1d observed in freshly isolated tissues, epithelium-derived cell lines, such as T84 cells, express low levels of CD1d. The reasons for such differences remain unclear. In these studies, we hypothesized that extracellular components derived from the intestinal milieu exist that are capable of influ- 
encing the levels of CD1d expressed by IECs. The lack of such components under in vitro culture conditions could explain, therefore, the relatively low level of CD1d expression observed in model IEC lines. However, other than the cytokine IFN- $\gamma$, essentially nothing is known about what factors regulate CD1d expression on any cell type (10). We now report that incubation of intestinal epithelium-derived cell lines with soluble lumenal components of the human and mouse gastrointestinal tract results in upregulation of CD1d expression. Biochemical and functional characterization of this CD1d-inducing activity from mouse and human samples identified it to be soluble heat shock protein 110 (Hsp110). The data presented from these studies predict a novel function for Hsp110, indicate the ability of lumenal components to influence cell surface molecule expression on IECs, and provide important insight into the mechanisms of $\mathrm{CD} 1 \mathrm{~d}$ regulation in vivo and in vitro.

\section{Methods}

Antibodies. Two antibodies specific for human CD1d were used in these studies. D5 is a mouse mAb of the IgG2a isotype. This antibody was raised against a glutathione- $S$-transferase-CD1d fusion protein containing the $\alpha 1-\alpha 3$ domains of CD1d (kindly provided by Steven Balk, Beth Israel Deaconess Medical Center, Boston, Massachusetts, USA; ref. 11). The mAb 51.1.3 (mouse IgG2b) was raised against an Fc-fusion protein of CD1d (kindly provided by Steven Porcelli, Albert Einstein Medical School, New York, New York, USA; ref. 12). P2A4 is a mouse anti-human ICAM- $1 \mathrm{mAb}$ and was obtained from the Developmental Studies Hybridoma Bank (Iowa City, Iowa, USA). L243 is a mouse mAb specific to human MHC class II (American Type Culture Collection, Rockville, Maryland, USA). Polyclonal anti-Hsp110 antibodies were previously characterized (13).

In vitro cell culture. The colon-derived human epithelial cell lines T84, HT-29, and Caco-2 were grown in a 1:1 mixture of Dulbecco/Vogt modified Eagle's medium and Ham's F-12 medium, supplemented with 15 mM HEPES buffer ( $\mathrm{pH} 7.5$ ), $14 \mathrm{mM} \mathrm{NaHCO} 3,40$ $\mathrm{mg} / \mathrm{ml}$ penicillin, $8 \mathrm{mg} / \mathrm{ml}$ ampicillin, $90 \mathrm{mg} / \mathrm{ml} \mathrm{strep-}$ tomycin, and $6 \%$ FCS. Cultures were maintained at $37^{\circ} \mathrm{C}$ in a humidified incubator containing $5 \% \mathrm{CO}_{2}$. Cells were passaged every $7-14$ days with $0.1 \%$ trypsin and $0.9 \mathrm{mM}$ EDTA in calcium- and magnesium-free PBS. Where indicated, human microvascular endothelial cells were used as described previously (14).

Exposure of epithelial cells to lumen-derived factors. Confluent monolayers were incubated for varying periods of time with preparations of lumen-derived samples or indicated concentrations of purified recombinant Hsp110 (15) on the apical and/or basolateral surface, as indicated. Prior to their addition to cell cultures, all samples were sterilized by passing of their contents through a $0.22-\mu \mathrm{m}$ sterile filter. The protein concentration of each sample was also assessed by use of the Bio-Rad protein assay reagent in accor- dance with the manufacturer's instructions (Bio-Rad Laboratories Inc., Hercules, California, USA).

Preparative methods for purification and characterization of lumenal contents. Six volunteers donated approximately $40 \mathrm{~g}$ of human feces each. Individual samples were resuspended in $50 \mathrm{ml}$ of PBS. Insoluble particulate matter was sedimented by centrifugation at $13,000 \mathrm{~g}$ for 10 minutes at room temperature and subsequently discarded. Soluble components represented within the supernatant were further clarified by passage through a $0.22-\mu \mathrm{m}$ sterile filter. The filtrate was defined as the crude lumenal contents (LC) and was used as a starting point from which to proceed with purification. In order to remove LC factors of less than $10 \mathrm{kDa}$ in molecular weight, crude samples were dialyzed against 100 times their volume of PBS for a period of 4 hours at $4{ }^{\circ} \mathrm{C}$. Molecules over $10 \mathrm{kDa}$ were retained within the confinement of the dialysis tube and were used in subsequent stages of the fractionation procedure. Aqueous and hydrophobic factors from LC samples were resolved by chloroform-induced phase separation. Briefly, 1 volume of chloroform was added to an equal volume of LC material. The mixture was vortexed for 10 minutes, and phases were resolved by centrifugation at $20,000 \mathrm{~g}$ for 20 minutes at room temperature. The upper phase comprised those molecules that partitioned to the aqueous solution and could be incubated with epithelial cells without the need for buffer exchange. The lower phase, containing hydrophobic factors, was dried and resuspended in PBS prior to subsequent use. To fractionate $\mathrm{LC}$ material on the basis of size, samples were passed under pressure through filters of varying molecular weights, ranging from 100 $\mathrm{kDa}$ to $10 \mathrm{kDa}$. Both filtrate and retentate from filtration procedures were applied to epithelial monolayers to assess elevations in CD1d expression.

Peptide microsequencing. In subsets of experiments, partially purified fractions of LC were resolved by SDSPAGE and stained by the Coomassie method, and the resulting approximately $100-\mathrm{kDa}$ band $(\sim 100 \mathrm{pmol})$ was extracted and submitted to the Dana-Farber Cancer Institute Peptide Core Facility (Boston, Massachusetts, USA) for trypsin digestion and microsequence analysis, using previously described protocols (16).

Preparation of intestine-derived material from mice. Groups of 6- to 12-week-old female mice of the BALB/c strain, bred under either specific pathogen-free (SPF) or germfree conditions, were used as previously described (17). All mice were sacrificed by asphyxiation in a carbon dioxide-saturated chamber followed by cervical dislocation. Lumenal components were then isolated from the stomach and the small and large intestine. Mucosal scrapings, representing the mucin-rich epithelial mucosa, were also isolated from the small and large intestine. Both mucous material and lumenal material were suspended in PBS (pH 7.4) and tested for their ability to upregulate CD1d on epithelial monolayers.

ELISA. Expression of epithelial cell surface proteins was evaluated by use of a cell surface ELISA, as previ- 
ously described on 96-well plates (18) or on permeable supports (19). The anti-CD1d antibody 51.1.3 was used at a final concentration of $30 \mu \mathrm{g} / \mathrm{ml}$ in HBSS containing $0.5 \%$ BSA. Antibodies specific to ICAM-1 (P2A4) and MHC class II (L243) were used as undiluted hybridoma supernatants. All incubations were performed at $4{ }^{\circ} \mathrm{C}$ for a period of 2 hours. Control samples were stained with isotype-matched antibodies and the secondary peroxidase conjugate. Data are presented as mean ODs at $405 \mathrm{~nm}$ blanked against values obtained from isotype-matched control samples. Variations between samples treated under one condition are represented by SEM.

Analysis of $m R N A$ levels by RT-PCR. Epithelial cells were lysed in Trizol reagent (GIBCO BRL; Life Technologies Inc., Rockville, Maryland, USA), and total cell RNA was purified according to the manufacturer's instructions. RNA samples were then treated with DNase I (GenHunter Corp., Nashville, Tennessee, USA) to remove any contaminating DNA. Reverse transcription was performed using a $\mathrm{CDNA}$ synthesis kit from $\mathrm{BD}$ Biosciences Clontech (Palo Alto, California, USA). Briefly, $1 \mu \mathrm{g}$ of total RNA was added to a reaction mixture of $50 \mathrm{mM}$ Tris- $\mathrm{HCl}$ ( $\mathrm{pH}$ 8.3), $75 \mathrm{mM} \mathrm{KCl}, 3 \mathrm{mM} \mathrm{MgCl}, 10 \mu \mathrm{M}$ of each dNTP, 20 pmol oligo-(dT)18 primer, 20 IU RNase inhibitor, and $200 \mathrm{IU}$ Moloney murine leukemia virus reverse transcriptase in a final volume of $20 \mu \mathrm{l}$. Singlestranded cDNA was synthesized using a Thermocycler (model PTC-200; MJ Research Inc.) set to perform one cycle at $42^{\circ} \mathrm{C}$ for 60 minutes followed by a second cycle at $94^{\circ} \mathrm{C}$ for 5 minutes. Amplification of CD1d-encoding transcripts by PCR was performed as described previously (10) using the Platinum Taq High Fidelity enzyme mix containing $1 \mu \mathrm{M}$ each of the CD1d-specific sense primer $\left(5^{\prime}\right.$-TTATCGAAGCAGCTTCACCAGG-3') and the antisense primer ( $5^{\prime}$-GATACCATGTCTCGTCAGCATT- $\left.3^{\prime}\right)$. Amplification reactions consisted of a 2-minute denaturation step at $94^{\circ} \mathrm{C}$ and a 1-minute annealing step at $60^{\circ} \mathrm{C}$, followed by repeated cycles of $68^{\circ} \mathrm{C}$ for 1 minute, $94^{\circ} \mathrm{C}$ for 45 seconds, and $60^{\circ} \mathrm{C}$ for 1 minute. At the end of the reaction, a final extension step was performed at $72^{\circ} \mathrm{C}$ for 10 minutes. Two rounds of amplification were required to detect $\mathrm{CD} 1 \mathrm{~d}$-encoding transcripts, as previously described (10). The first round consisted of 25 cycles, and the second round consisted of 15 cycles. Products of PCR were resolved on a $1 \%$ agarose gel containing ethidium bromide. A 518-bp fragment corresponding to CD1d transcript expression was visualized under ultraviolet light. To ensure that equal quantities of template had been loaded between samples, the housekeeping gene GAPDH was amplified using $1 \mu \mathrm{M}$ of the gene-specific primer pair purchased from CLONTECH Laboratories Inc. GAPDH fragments appeared as a 983-bp band when resolved by electrophoresis on a $1 \%$ agarose gel.

Immunoprecipitation and Western blot analysis. Following experimental conditions, epithelial monolayers were washed with ice-cold HBSS and lysed in $500 \mathrm{ml}$ of RIPA buffer consisting of $50 \mathrm{mM}$ Tris- $\mathrm{HCl}(\mathrm{pH} 7.4), 150 \mathrm{mM}$ $\mathrm{NaCl}, 1 \% \mathrm{NP}-40,0.5 \%$ sodium deoxycholate, $0.1 \% \mathrm{SDS}$,
$500 \mathrm{mM}$ phenylmethanesulfonyl fluoride, and 1 $\mathrm{mg} / \mathrm{ml}$ each of pepstatin, aprotinin, and leupeptin. Insoluble material was removed by centrifugation at $13,000 \mathrm{~g}$ for 10 minutes at $4^{\circ} \mathrm{C}$. Supernatants, normalized for protein concentration, were precleared by rotation with normal mouse serum and protein A-conjugated Sepharose beads. CD1d from cell lysates was precipitated with $20 \mu \mathrm{g}$ of either D5 or 51.1.3 bound to protein $\mathrm{A}-S e p h a r o s e$. Incubations with $\mathrm{CD} 1 \mathrm{~d}$-specific antibodies were performed overnight at $4^{\circ} \mathrm{C}$. Immunoprecipitates were washed with RIPA buffer, boiled in $1 \times$ reducing Laemmli buffer, and resolved by $12 \%$ SDSPAGE. Proteins were transferred to nitrocellulose and blocked overnight in PBS containing 5\% nonfat dry milk and $0.05 \%$ Tween-20. Nitrocellulose blots were then incubated for 1 hour with D5 antibody $(4 \mu \mathrm{g} / \mathrm{ml})$ followed by species-matched peroxidase-conjugated IgG $(1 \mu \mathrm{g} / \mathrm{ml})$ for 45 minutes. In subsets of experiments, Hsp110 in LC was determined by Western blot using rabbit polyclonal antisera, as previously described (13). HRP detection on nitrocellulose blots was achieved using ECL.

Localization of Hsp110 in human tissue. Cryopreserved human large and small intestinal tissue samples were obtained from the tissue bank of the Brigham and Women's Hospital and Massachusetts General Hospital. Samples were frozen in OCT compound (Bayer, Elkhart, Indiana, USA) and stored at $-80^{\circ} \mathrm{C}$ until use. Four-micrometer-thick frozen sections were air-dried for 20 minutes and stained by the avidin-biotin complex method as previously described (20). Briefly, the sections were fixed in acetone for 10 minutes, air-dried, and incubated with purified rabbit anti-human Hsp110 polyclonal antibody at 1:100 dilution for 1 hour. As a negative control, parallel sections were incubated with $2 \%$ rabbit serum. After incubation, specimens were incubated for 30 minutes with $0.3 \%$ hydrogen peroxide in PBS to block endogenous peroxidase activity. Endogenous biotin was blocked by sequential incubations with avidin (Vector Laboratories Inc., Burlingame, California, USA) and biotin (Sigma-Aldrich, St. Louis, Missouri, USA). Specimens were then incubated with biotinylated goat anti-rabbit Ig (Vector Laboratories Inc.) at 1:200 dilution followed by avidin-biotin HRP complex (DAKO Corp., Carpinteria, California, USA). After washing, the specimens were developed in a solution of 3-amino-9-ethylcarbazole (Aldrich Chemical Co., Milwaukee, Wisconsin, USA). After developing, specimens were counterstained with hematoxylin and mounted with Glycergel (DAKO Corp.).

In subsets of experiments, Hsp110 was localized to the surface of primary culture enterocytes. Briefly, enterocytes were isolated by a method described previously (21). Surgical specimens were obtained from the operating room. Specimens were washed extensively with PBS containing $1 \%$ penicillin/streptomycin and $1 \%$ Fungizone (Flow Laboratories, McLean, Virginia, USA). The mucosa was stripped from the submucosa, minced, and placed in $1 \mathrm{mM}$ dithiothreitol (Sigma-Aldrich) for 
5 minutes at room temperature to remove the mucus. The pieces were then washed in PBS and incubated in dispase $(3 \mathrm{mg} / \mathrm{ml}$ in RPMI 1640 medium; Roche Applied Science, Indianapolis, Indiana, USA) for 30 minutes in a $37^{\circ} \mathrm{C}$ shaking incubator. This was repeated four times. The tissue pieces were removed and the cell suspension collected, pooled, and centrifuged on a Percoll density gradient (Amersham Biosciences Corp., Piscataway, New Jersey, USA). Enterocytes located at the 0-30\% interface were washed three times with PBS and resuspended in RPMI medium with $0.1 \%$ BSA. Preparations of purified enterocytes were greater than $90 \%$ viable, free of macrophages and B cell contamination as determined by staining with anti-CD14 and anti-CD20 mAb's (Coulter Corp., Hialeah, Florida, USA), and contaminated with only $2-4 \%$ intraepithelial lymphocytes $\left(\mathrm{CD}^{+}\right.$cells). Enterocytes were stained with anti-Hsp110 (1:100 dilution of rabbit polyclonal) or an equivalent dilution of normal rabbit serum, followed by FITClabeled goat anti-rabbit secondary antibody. Stained enterocytes were analyzed on an EPICS Profile III flow cytometer (Beckman Coulter Inc., Fullerton, California, USA), as described previously (21).

\section{Results}

Soluble lumenal components induce expression of CD1d on IECs. Monolayers of epithelial cells form a selective barrier between the lumen of the intestine and the underlying intestinal mucosa. As a consequence, IECs are constantly exposed to the lumenal environment and its contents (22). To assess whether such exposure could result in alterations in epithelial CD1d expression, T84 cells were cultured for varying periods of time with soluble LC prepared as described and diluted in media to final protein concentrations of $3 \mu \mathrm{g} / \mathrm{ml}$ and $6 \mu \mathrm{g} / \mathrm{ml}$. Figure 1a depicts cell surface ELISA using the CD1dspecific antibody 51.1.3. OD values at $405 \mathrm{~nm}$ reflect the degree of HRP-bound secondary antibody association with 51.1.3 and therefore represent the relative levels of cell surface CD1d expression. Of note, since basolateral access is limited with cells grown on plastic (19), these assays likely reflect a predominant apical CD1d signal. Exposure of T84 cells to LC on the apical surface resulted in a time-dependent increase in the level of cell surface CD1d expression, peaking by 48 hours of exposure. The degree of surface CD1d upregulation was also dependent on the concentration of LC material present in cultures, being significantly higher when cells were exposed to $6 \mu \mathrm{g} / \mathrm{ml}(\mathrm{OD}=0.45 \pm 0.029)$ rather than $3 \mu \mathrm{g} / \mathrm{ml}(\mathrm{OD}=0.27 \pm 0.032)$, compared with T84 cells incubated alone without exposure to LC material $(\mathrm{OD}=0.05 \pm 0.015, n=6$ at 48 hours, $P<0.01$ by ANOVA in comparison to $3 \mu \mathrm{g} / \mathrm{ml} / \mathrm{LC}$ and $6 \mu \mathrm{g} / \mathrm{ml}$ samples). The upregulation of CD1d by lumenal material was observed with all LC samples analyzed, including those from subjects with inflammatory bowel disease (data not shown). As a control for the induction of CD1d expression, cells were cultured in the presence of IFN- $\gamma$ for 48 hours at a concentration of $1,000 \mathrm{IU} / \mathrm{ml}$.
As in previous studies (10), IFN- $\gamma$ elevated levels of CD1d on the surface of T84 cells (OD $=0.32 \pm 0.042$, $P<0.01$ compared with no IFN- $\gamma$ ). To determine whether the effects of LC components on epithelial cell phenotype were specific to CD1d, levels of cell surface MHC class II and ICAM-1 were also assessed by ELISA (Figure $1 \mathrm{~b}$ ). Upon exposure to IFN- $\gamma$ at $1,000 \mathrm{U} / \mathrm{ml}$ for 48 hours, T84 cells displayed elevated levels of both MHC class II and ICAM-1 expression. In contrast, however, no significant alterations in the surface expression levels of these two markers were detected in cultures treated with $6 \mu \mathrm{g} / \mathrm{ml} \mathrm{LC}$ material over the same time period. These results demonstrate that upregulation of CD1d by $\mathrm{LC}$ is relatively specific.

To determine whether the LC response might be polarized, we subjected T84 cells to LC exposure on the apical or basolateral surface and assessed CD1d expression by ELISA. To do this, T84 cells were grown to electrical confluence (transepithelial resistance $>1,000$ ohms $\times \mathrm{cm}^{2}$ ) on permeable supports, and ELISA was performed as described previously (19). Interestingly, some degree of polarity was apparent. While apical exposure to $\mathrm{LC}$ resulted in a greater than fourfold induction of CD $1 \mathrm{~d}(\mathrm{OD}=0.37 \pm 0.011$ with LC compared with $\mathrm{OD}=0.08 \pm 0.01$ without $\mathrm{LC}, n=5$ at 48 hours, $P<0.01$ by ANOVA), a less than twofold change was observed when LC was delivered to the basolateral surface $(\mathrm{OD}=0.15 \pm 0.005$ with $\mathrm{LC}$ compared with $\mathrm{OD}=0.08 \pm 0.01$ without LC, $n=5$ at 48 hours, $P<0.05$ by ANOVA). Such findings suggest that a degree of polarity may exist in the LC response.

ELISA data shown in Figure 1 revealed the increased levels of cell surface CD1d expression following exposure of IECs to LC. To determine whether elevations in surface CD1d levels were accompanied by an overall increase in CD1d transcription, CD1d mRNA levels
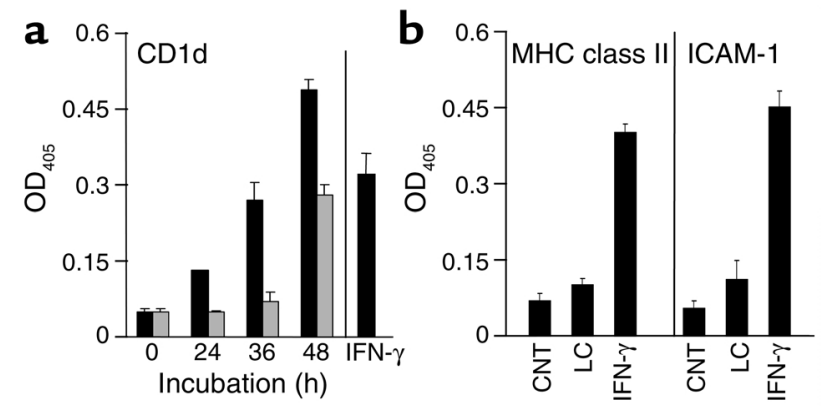

\section{Figure 1}

Soluble components present within human LC selectively enhance CD1d expression on the surface of IECs. (a) Cell surface ELISA of T84 monolayers following incubation with either $6 \mu \mathrm{g} / \mathrm{ml}$ (black bars) or $3 \mu \mathrm{g} / \mathrm{ml}$ (gray bars) of LC sample for varying periods of time. (b) Relative levels of CD1d, MHC class II, and ICAM-1 expression were compared by ELISA on T84 cells incubated alone (CNT) or in the presence of $6 \mu \mathrm{g} / \mathrm{ml}$ LC sample (LC) for a period of 48 hours. As a positive control for the detection of all inducible epithelial markers, monolayers were exposed to IFN- $\gamma$ for 48 hours at a final concentration of $1,000 \mathrm{IU} / \mathrm{ml}$. ODs at $405 \mathrm{~nm} \pm \mathrm{SEM}$, generated by ELISA, were blanked against isotype-matched control antibodies. 


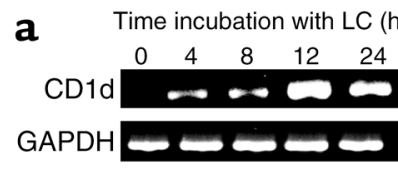

c
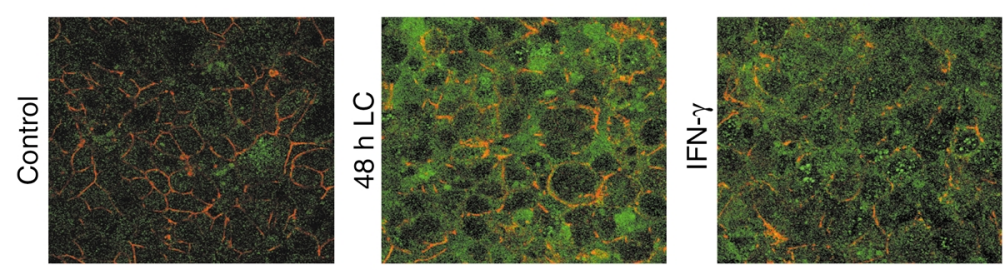

\section{Figure 2}

LC-induced upregulation of cell surface CD1d expression is accompanied by an elevation in the levels of CD1d-encoded mRNA transcripts and protein. (a) RT-PCR of total RNA isolated from T84 cells treated for varying lengths of time with media containing $6 \mu \mathrm{g} / \mathrm{ml}$ human LC sample. Primers specific to CD1d and the housekeeping gene GAPDH were used as described. (b) Immunoprecipitation (IP) of whole-cell lysates following treatment with LC material, using the CD1d-specific antibodies D5 and 51.1.3. Immunoprecipitates were resolved by $12 \%$ SDS-PAGE under nonreducing conditions. Proteins were transferred to nitrocellulose, and CD1d was detected by immunoblotting with the D5 antibody. Locations of $48-\mathrm{kDa}$ (filled arrowhead) and $37-\mathrm{kDa}$ (open arrowhead) forms of CD1d are indicated. The molecular weight is indicated on the left. (c) Confocal microscopy of T84 cells following incubation for 48 hours alone or in the presence of LC sample $(6 \mu \mathrm{g} / \mathrm{ml})$ or IFN- $\gamma(1,000 \mathrm{IU} / \mathrm{ml})$. CD1d (green) and the tight-junction marker ZO-1 (red) were stained with FITC- and Texas red-conjugated antibodies, respectively.

were analyzed by RT-PCR. As shown in Figure 2a, exposure of T84 cells to $6 \mu \mathrm{g} / \mathrm{ml} \mathrm{LC}$ resulted in an increase in the level of mRNA encoded by the CD1d gene. Increased mRNA levels were detectable by 4 hours of cell culture and reached maximal levels by 12 hours. Equal loading of total cell RNA was confirmed by parallel examination of the housekeeping gene GAPDH. Detection of CD1d at the protein level was then assessed by immunoprecipitation of CD1d from wholecell lysates using the antibodies D5 and 51.1.3. Contents of immunoprecipitates were resolved by SDS-PAGE and transferred to nitrocellulose, and CD1d was detected by probing of membranes with the D5 antibody. The results of immunoprecipitation using D5 are shown in the left panel of Figure 2b. T84 cells, which had been stably transfected with the cDNA-encoding CD1d (T84d), have been previously characterized and are known to express significantly higher levels of CD1d than untransfected cells (8). Extracts from T84d cells were used as a positive control. Blots of the proteins immunoprecipitated from T84d cells with the D5 antibody revealed two major bands. The $37-\mathrm{kDa}$ (open arrowhead) and 48-kDa (filled arrowhead) bands have been previously characterized and have been shown to correlate with nonglycosylated and glycosylated forms, respectively $(9,11,23)$. After immunoblotting with the D5 antibody, immunoprecipitated extracts of T84 cells cultured in media alone revealed low levels of these two CD1d protein bands. Incubation of cells with LC for 48 hours resulted in the detection of increasing amounts of the 37- and 48$\mathrm{kDa}$ forms of CD1d as defined by $\mathrm{D} 5$ immunoprecipitation and Western blotting. By 48-72 hours of culture with soluble LC, the levels of CD1d protein detected were similar to that detected in the T84d samples. Immunoprecipitation with 51.1.3, which primarily recognizes mature, fully glycosylated CD1d (11), also revealed an increase in the level of the $48-\mathrm{kDa}$ form of CD1d (Figure $2 \mathrm{~b}$, right panel, filled arrowhead).

Characterization of CD1d protein expression was also assessed, using confocal microscopy (Figure 2c). T84 cells grown to confluence and cultivated under varying conditions were permeabilized and stained with a combination of the 51.1.3 antibody and an antibody specific for the tight-junction protein ZO-1. Incubation of cells with isotype-matched antibody controls resulted in no specific staining (data not shown). Under all conditions, cell outlines were distinguished by $\mathrm{ZO}-1$ border staining. Cells cultivated in the presence of media alone displayed a low degree of CD1d staining (Figure $2 c$, left panel), while culture of cells with IFN- $\gamma(1,000 \mathrm{U} / \mathrm{ml})$ for 48 hours resulted in increased levels of CD1d observed throughout cell cross sections (Figure 2c, right panel). CD1d-specific staining of IFN- $\gamma$-treated samples appeared granular and diffuse throughout the monolayer. Similarly, exposure of T84 cells to LC material for 48 hours resulted in a significant increase in the degree of CD1d-specific staining above that observed in control monolayers. Taken together, the results illustrated in Figures 1 and 2 demonstrate the ability of soluble components present within LC to upregulate CD1d protein and mRNA expression. Elevated levels of CD1d by LC are comparable to the levels observed in cells treated with IFN- $\gamma$ or transfected with a construct containing the CD1d gene and controlled with an exogenous promoter (8). LC-induced CD1d is not limited to T84 cells. To establish that the effects of LC on CD1d expression were not specific to the T84 cell line, levels of CD1d were examined on other epithelium-derived lines, namely HT-29 and Caco-2, following exposure to LC material. As shown by ELISA in Figure 3a, culture of HT-29 cells with LC material at a concentration of $6 \mu \mathrm{g} / \mathrm{ml}$ resulted in a time-dependent increase in cell surface CD1d expression. As with T84 cells, CD1d expression reached maximal levels by 48 hours of culture and was quantitatively comparable to the levels observed in samples treated with $1,000 \mathrm{U} / \mathrm{ml}$ IFN- $\gamma$.

In subsets of experiments, we also examined whether LC induced CD1d on cell types other than epithelia. 


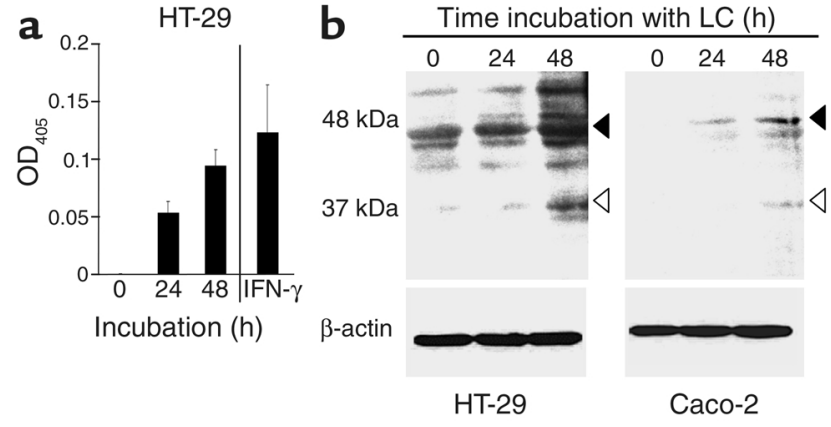

Figure 3

Soluble LC material elevates CD1d expression in the colonic epithelium-derived cell lines HT-29 and Caco-2. (a) CD1d-specific cell surface ELISA of confluent monolayers of HT-29 cells following incubation with LC samples. As a positive control, cells were exposed to IFN- $\gamma$ at a final concentration of $1,000 \mathrm{IU} / \mathrm{ml}$ for a period of 48 hours. (b) Western blot of whole-cell lysates isolated from HT-29 and Caco-2 cells following treatment with soluble LC material at a concentration of $6 \mu \mathrm{g} / \mathrm{ml}$. Proteins contained within the lysates were resolved by $12 \%$ SDS-PAGE and transferred to nitrocellulose. The presence of CD1d was subsequently assessed by probing with the anti-human CD1d-specific antibody D5. CD1d was observed as dominant bands of 48-kDa (filled arrowhead) and 37-kDa (open arrowhead) forms. No bands were detected when nitrocellulose membranes were probed with normal mouse serum (data not shown). The blots were reprobed for the presence of $\beta$-actin (bottom panels).

Specifically, we addressed whether microvascular endothelial CD1d might be regulated by LC. Such experiments revealed that while IFN $-\gamma(300 \mathrm{U} / \mathrm{ml}, 48$ hours) increased CD1d on the surface of microvascular endothelia $(2.5 \pm 0.6$-fold increase in OD by ELISA, $P<0.01$ compared with no IFN- $\gamma$ ), LC did not influence endothelial CD1d $(0.3 \pm 0.2$-fold increase in OD by ELISA, $P=$ not significant compared with no IFN- $\gamma$ ).

Cell surface ELISA of Caco- 2 cells was not performed, since adherence of epithelial monolayers proved insufficient to withstand extensive washing necessary for the procedure (data not shown). Therefore, whole-cell CD1d levels were next examined for both HT-29 and Caco-2 cultures by probing of lysates resolved by Western blot. Immunoblots of extracts obtained from HT-29 cells cultivated in media alone revealed the presence of two bands of approximately $46-48 \mathrm{kDa}$ when probed with the D5 antibody. Following exposure of HT-29 cells to LC material for 24-48 hours, a band of $37 \mathrm{kDa}$ and increased intensity of the $46-$ to $48-\mathrm{kDa}$ bands were detected in the cell extracts. Of note, and as we have previously reported (11), whereas the 51.1.3 antibody recognizes mature $\mathrm{CD} 1 \mathrm{~d}$, the $\mathrm{D} 5 \mathrm{mAb}$ preferentially recognizes immature $C D 1 d$ that has not yet acquired maturation of the carbohydrate side-chain modifications. Therefore, it is likely that the middle bands observed within the D5 Western blot represent immature CD1d with partially processed carbohydrate side-chain modifications. The migration pattern of these inducible bands matched the patterns observed in the immunoblots of T84d extracts (data not shown) and presumably represents forms of CD1d that vary in their degree of glycosylation. Immunoblots of lysates obtained from Caco-2 cells also revealed the induction of CD1d-specific bands following exposure to LC material. As with the T84 and HT-29 cells, a band of $37 \mathrm{kDa}$ and bands between 46 and $48 \mathrm{kDa}$ became readily visible after 48 hours of cultivation in the presence of soluble LC. In both cases, $\beta$-actin Western blotting was used to demonstrate equal loading (Figure $3 b$ ). Thus, such LC-induced upregulation of CD1d expression was not specific for T84 cells but may be rather specific for intestinal epithelia.

Non-microbe-derived, heat-labile components isolated from the lumen of the murine intestinal tract stimulate CD1d upregulation on the surface of T84 cells. To better define the origin of CD1d-inducing activity in LC, soluble LC were isolated from the intestinal tract of mice and tested for their influence on CD1d expression by T84 cell ELISA. Incubation of T84 cells with $6 \mu \mathrm{g} / \mathrm{ml}$ lumenal extracts obtained from mice housed under SPF conditions resulted in the induction of CD1d cell surface expression by 48 hours of culture (Figure 4a). To determine whether the murine CD1d-inducing factor was derived from components of the microbial flora, lumenal samples isolated from mice bred under germfree conditions were incubated with T84 cells. Exposure of T84 cells to germfree lumenal extracts led to elevations in surface CD1d levels that did not differ significantly from the levels induced by SPF-derived lumenal samples. These data demonstrate that lumenal extracts devoid of microbial content are capable of upregulating CD1d expression on

\section{Figure 4}

Non-microbe-derived factors are present within the lumen of the murine small and large intestine that upregulate CD1d expression on human T84 cells. Cell surface ELISA was performed on confluent T84 monolayers using the CD1d-specific antibody 51.1.3. (a) BALB/c mice housed in either germfree (GF) or SPF environments were sacrificed and the LC of the colon isolated. T84 cells were cultured alone (-) or exposed to the soluble lumenal preparations for 48 hours at a final concentration of $6 \mu \mathrm{g} / \mathrm{ml}$. (b) Lumenal material from the stomach (St), small intestine (SI), or large intestine (LI) of SPF-housed mice was tested for its ability to enhance epithelial cell surface CD1d expression. The CD1d-inducing activity of both soluble lumenal components (black bars) and mucosal scrapings (gray bars) was assessed relative to untreated controls $(-)$.

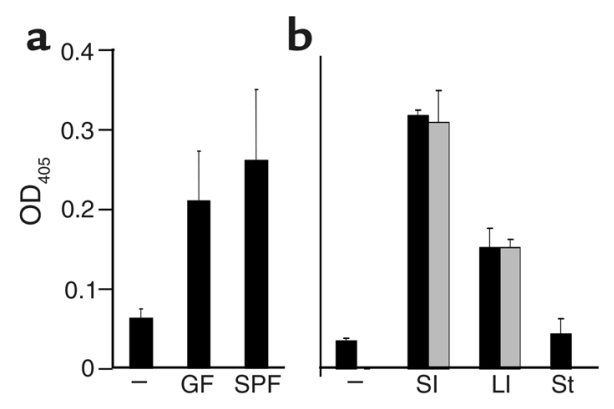

Fraction 

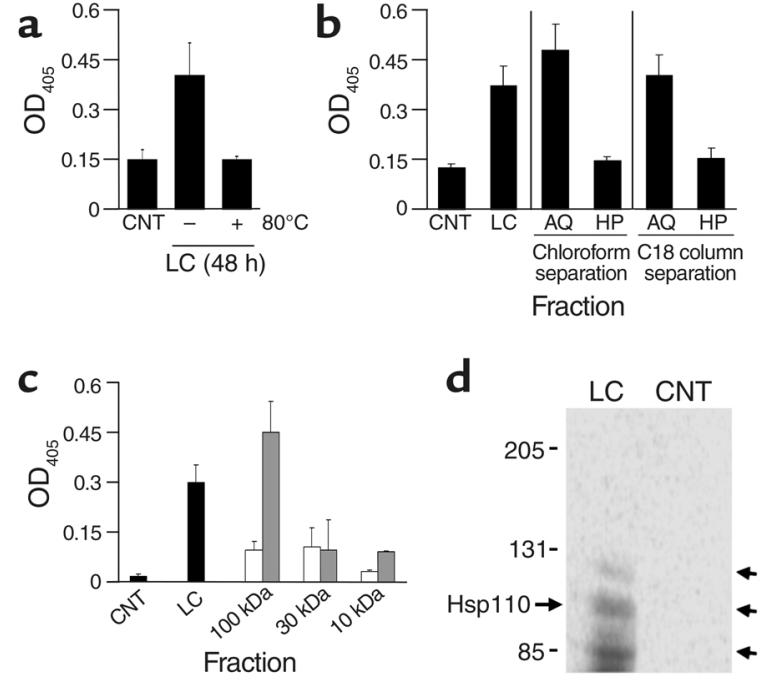

\section{Figure 5}

Preliminary biochemical characterization of the active CD1d-inducing factor present within soluble LC material. ELISA was performed on T84 cells treated for a period of 48 hours with various preparations of LC isolates. (a) Monolayers were cultured alone (CNT), in the presence of unmodified whole LC samples (-), or with LC samples that had been preincubated at $80^{\circ} \mathrm{C}$ for 10 minutes. (b) LC material was separated into aqueous (AQ) or hydrophobic (HP) phase by chloroform extraction and C18 column chromatography, respectively, as indicated. Individual fractions were subsequently assessed for their ability to induce CD1d expression. (c) LC samples were passed through a Centricon filter (Millipore Corp., Bedford, Massachusetts, USA) of 100-kDa molecular weight cut-off. The $100-k D a$ filtrate was then passed consecutively through $30-\mathrm{kDa}$ and $10-\mathrm{kDa}$ Centricon filters. The filtrates (white bars) and retentates (gray bars) from each filtration step were subsequently resuspended in PBS, tested for their CD1d-inducing activity (black bars), and compared with those from the no-treatment (CNT) and crude-fraction (LC) groups. (d) Coomassie-stained SDS-PAGE gel of partially purified material, indicating the major bands selected for microsequencing (arrows), and the migration position of the band with sequence homology to Hsp110. A control (CNT) derived from a100-kDa filtrate fraction is also shown.

epithelial cells, implicating a non-microbe-derived component as the active CD1d-inducing factor.

It is also possible that LPS contributes to this CD1dinducing activity, even in germfree animals (e.g., LPS in autoclaved food, water, etc.). Therefore, we examined whether LPS might induce CD1d. T84 cells exposed to LPS (derived from Salmonella minnesota; range 0.01-100 $\mathrm{ng} / \mathrm{ml}$ in the presence of $5 \%$ normal human serum) resulted in no change in CD1d expression compared with controls (maximal increase $0.2 \pm 0.1$-fold, $P=$ not significant). As controls, monolayers treated in a similar fashion and assessed for ICAM-1 induction resulted in a rapid and concentration-dependent elevation in ICAM-1 surface expression (maximal increase $18 \pm 0.8$-fold, $P<0.01)$. Such findings demonstrate that, while epithelia can respond to LPS, it is unlikely that CD1d-inducing activity in LC represents LPS.

To establish the region of the gastrointestinal tract displaying the highest degree of CD1d-inducing activ- ity, lumenal samples and mucosal scrapings were isolated from the small intestine, the large intestine, and the stomach. Figure $4 \mathrm{~b}$ reveals that induction of CD1d expression on T84 cells was greatest when cells were incubated with mucosal and lumenal extracts from the small intestine. Upregulation of CD1d was clearly detectable in cultures containing large bowel-derived samples, but not across monolayers cultured in the presence of stomach-derived material. These data demonstrate that the active CD1d-inducing component is present both along the mucous layer lining the epithelium and in the lumen of the gut itself. Maximum activity is present within regions of the proximal small intestine. In contrast, no activity could be detected in contents obtained from the stomach.

Identification of Hsp 110 as the CD1d-inducing activity. To begin to characterize the active component within LC responsible for induction of CD1d expression, we initially examined heat stability, hydrophobicity, and nominal molecular size of the active LC component(s). To determine whether the activity was heat sensitive, soluble LC components were incubated at $80^{\circ} \mathrm{C}$ for 10 minutes and then exposed to cell monolayers. In contrast to unmodified LC material, heat-treated samples did not elicit a detectable elevation in CD1d expression (Figure 5a). Hydrophobicity of the active LC component was determined by chloroform extraction or by C18 column chromatography. For both separation techniques, the CD1d-inducing activity partitioned into the aqueous phase (Figure $5 \mathrm{~b}$ ). Molecular weight fractionation was performed using the upper aqueous phase of chloroform extracts as starting material. This fraction was passed through a series of consecutively lower-molecular weight cut-off filters. Retentates and filtrates from each filtration procedure were subsequently tested on T84 cell monolayers for their ability to stimulate CD1d expression. As indicated in Figure $5 \mathrm{c}$, the retentate generated from filtration using the $100-\mathrm{kDa}$ filter possessed the vast majority of activity, as reflected by the ability of this fraction to upregulate CD1d expression to a level comparable to that for whole untreated LC material. The $100-\mathrm{kDa}$ filtrate and the fractions generated by its filtration through lower-molecular weight filters displayed very little CD1d-inducing activity. Identical results were obtained with murine LC fractions from both SPF and germfree mice (data not shown).

Using partially purified extract (aqueous phase following chloroform extraction eluted from C18 chromatography with molecular weight $>100 \mathrm{kDa}$ ), SDSPAGE analysis revealed three distinct bands of $M_{r} 85$, 110 , and $125 \mathrm{kDa}$ by Coomassie staining (Figure 5d). No specific sequence was obtained from the 85 - or 125 $\mathrm{kDa}$ bands. Microsequence analysis of tryptic peptides from the approximately $110-\mathrm{kDa}$ protein revealed three peptides (sequences IEVPLHSLMEQTHLK, ELNNVCEPVVNQPKPK, and KPVTDCVISVPSFFTDAER) with direct sequence homology to Hsp110 (15). As shown in Figure 6a, Western blot analysis of unfrac- 
tionated human LC material revealed the presence of an antigen consistent with purified Hsp110, with slightly slower mobility by SDS-PAGE, likely related to an as-yet undetermined posttranscriptional and/or posttranslational modification. Similar analysis with primary antibody omitted revealed no detectable signal. Taken together, these studies suggest the presence of relatively high concentrations of Hsp110 in our LC fractions.

As proof of principle for Hsp110-mediated induction of CD1d, two antibody-antigen approaches were used. First, as shown in Figure 6b, the addition of increasing concentrations of anti-Hsp110, but not control anti-Hsp30, to LC extracts resulted in decreased CD1d-inducing activity in a concentrationdependent fashion $(P<0.025$ by ANOVA). Second, as shown in Figure $6 c$, the addition of purified mammalian Hsp110 to epithelial monolayers resulted in a concentration-dependent induction of CD1d by surface ELISA $(P<0.025$ by ANOVA). The addition of anti-Hsp110, but not anti-Hsp30, significantly diminished CD1d-inducing activity of purified Hsp110 protein $(P<0.05)$. Taken together, these data identify Hsp110 as a component of the LC extract, presumably derived from the IEC itself, in the autocrine regulation of epithelial CD1d cell surface expression.

Finally, given the nonmicrobial origin of Hsp110 (Figure 4a) consistent with the absence of known prokaryotic Hsp110 isoforms (15), and the relative abundance of CD1d-inducing activity in the small intestine (Figure 4b), we questioned the origin of Hsp110 within LC. First, we addressed whether Hsp110 was detectable on native enterocytes. As shown in Figure 6d, flow cytometric analysis of native small intestine enterocytes revealed dominant Hsp110 surface expression. Interestingly, exposure of native epithelia to recombinant Hsp110 (10 $\mu \mathrm{g} / \mathrm{ml}, 24$ hours $)$ did not induce CD1d expression above base line (data not shown), suggesting that in the native state, this signaling pathway may already be saturated. To morphologically confirm these findings, immunohistochemistry was performed on normal small intestine $(n=3)$ and large intestine $(n=2)$ tissue samples (Figures 6, e and f, respectively). These studies revealed abundant expression of Hsp110 in small intestinal samples, with prominent localization to the epithelium and patchy staining within the lamina propria. Hsp110 expression within large intestinal epithelia was also evident, although less prominent than in the small intestine (Figure 6f). Taken together, these findings indicate that the epithelium is a rich source of Hsp110 in the intestine and, as such, likely reflects the CD1d-inducing activity evident in LC.

\section{Discussion}

The ability of CD1d to stimulate immune responses that regulate intestinal inflammation implicates this molecule as an important component of the mucosal immune system (8). Characterization of the events that regulate $\mathrm{CD} 1 \mathrm{~d}$ expression in general, and on the surface of IECs in particular, promises to reveal further information as to the functional role of CD1d. Similar to other MHC class I-related molecules, CD1d expression and function are regulated by IFN- $\gamma(10)$. However, little is known about other endogenous pathways for such regulation. We demonstrate here that lumenal, IEC-derived Hsp110 represents a novel regulatory component for CD1d expression on epithelia.

A number of previous studies have suggested discordant in vivo and in vitro expression of CD1d; namely, that $\mathrm{CD} 1 \mathrm{~d}$ expression is higher in vivo than in in vitro
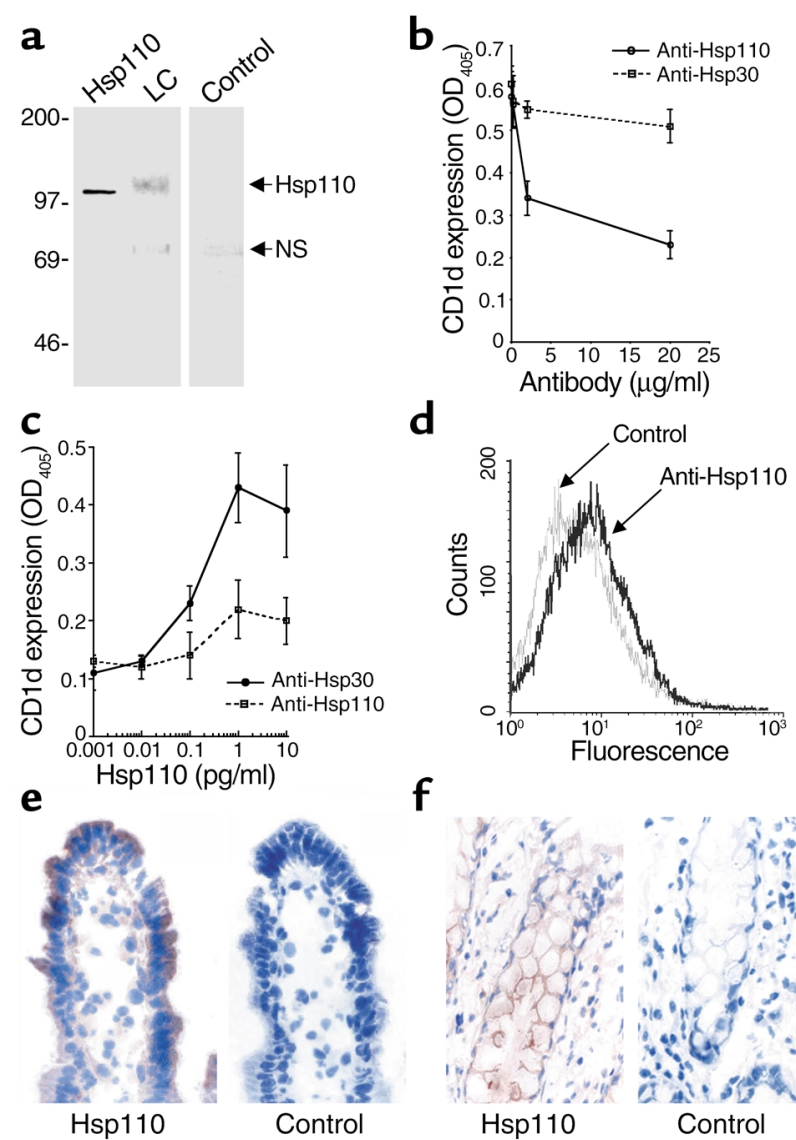

f

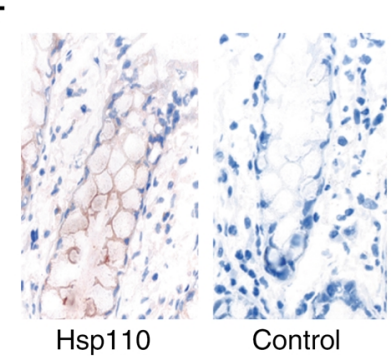

Figure 6

Role of Hsp110 in induction of epithelial CD1d. (a) Western blot analysis of LC for Hsp110. LC or purified recombinant Hsp110 (0.3 $\mu \mathrm{g})$ was resolved by SDS-PAGE under nonreducing conditions, and blots were probed with polyclonal rabbit anti-Hsp110. Also shown is the control with primary antibody omitted from the reaction mixture. A nonspecific band (NS) of approximately $70 \mathrm{kDa}$ was observed in both LC and control samples. (b) Cell surface ELISA of T84 monolayers following incubation with $6 \mu \mathrm{g} / \mathrm{ml} \mathrm{LC}$ in the presence of indicated concentrations of anti-Hsp110 or control anti-Hsp30. (c) Cell surface ELISA of T84 monolayers following incubation with indicated concentrations of purified recombinant Hsp110 in the presence of anti-Hsp110 or control anti-Hsp30. OD values generated by ELISA were blanked against isotype-matched control antibodies. (d) Flow cytometric analysis of Hsp110 on native small intestinal enterocytes (black line) compared with equivalent concentrations of an isotypematched control (gray line). (e and f) Immunohistochemical localization of Hsp110 in normal small intestinal tissue (e) and in normal colon (f). Positive staining with anti-Hsp110 but not with control Ig (Control) is seen in epithelial cells and diffusely in the lamina propria. Magnification, $\times 400$. 
culture systems. Such studies suggest the possibility that additional endogenous components may be necessary to induce and/or maintain surface levels of epithelial CD1d. As such, we screened human and murine LC for the existence of CD1d-inducing bioactivity. Such experiments revealed a prominent factor(s) that partitions into high-molecular weight, aqueous-phase components. A possibility was that LPS may represent the CD1d-inducing activity. However, four observations ruled out LPS. First, we directly demonstrated that LPS does not regulate CD1d expression on T84 epithelium. Second, LPS has been shown to induce ICAM-1 on IECs (24), and our results indicated that LC fail to regulate the expression of epithelial ICAM-1. Third, LC derived from both conventionally housed mice and germfree mice readily induced CD1d on human epithelia, likely indicating that this activity is not LPS but is of eukaryotic origin. Finally, the sensitivity of CD1d-inducing activity to heat denaturation argued against LPS activity, since LPS is heat stable. Using biochemical and biophysical properties of this bioactivity, we identified Hsp110 as an LC-derived agonist for CD1d expression. Subsequent studies revealed that epithelia from the normal human intestine prominently express Hsp110, and that purified Hsp110 can recapitulate the activity observed in crude LC, suggesting an autocrine pathway for Hsp110 function.

The mechanism(s) of CD1d induction by Hsp110 is not known. It is possible that Hsp110 binds directly to epithelial surface receptors and couples to intracellular signaling. While much attention has been paid to understanding the role of extracellular Hsp signaling in a variety of disease states (25), mechanisms or surface targets for Hsp's are only recently appreciated. Of distinct interest, several studies suggest that Toll-like receptors (TLRs) may represent one class of receptors for Hsp family members $(26,27)$ and that Hsp70 can function as a "cytokine-like" molecule in association with CD14 (28). We do not know whether Hsp110 binds to TLR-2 or TLR-4 or whether CD14 is necessary for CD1d-inducing activity. IEC lines are known to express functional TLRs $(29,30)$ and have been demonstrated to signal in a CD14-dependent manner (31). Similarly, since it was recently shown that CD40 is a receptor for the Hsp70 family (32), it is possible that CD40 provides a receptor for Hsp110. However, studies directed at this issue have revealed no detectable CD40 on intestinal epithelia (L. Mayer, unpublished observations). Another candidate molecule is CD91 (also called $\alpha_{2}$-macroglobulin receptor or the LDLrelated protein), as it has been demonstrated to bind directly to a number of Hsp's $(33,34)$. However, CD91 has not been shown to directly bind Hsp110, and little is known about CD91 expression on intestinal epithelia. Alternatively, it is possible that Hsp110 does not bind directly to an epithelial surface receptor. Thus it is possible that functional Hsp110 is in complex with an as-yet unidentified peptide(s) that activates CD1d via chaperone function and/or costimulation of other surface molecules. All Hsp's described to date have attributable chaperone function (25), raising the distinct possibility that such chaperone function provides an accessory signal for induction of CD1d.

At present, it is not known whether Hsp110-mediated regulation of $\mathrm{CD} 1 \mathrm{~d}$ represents a physiologic regulatory pathway or a process induced by immune activation. Given the constitutive presence of Hsp110 in the lumen of all individuals examined, it might be argued that Hsp110-mediated regulation of CD1d is a physiologic process. In the context of gut-associated lymphoid tissue, it could similarly be argued that Hsp 110 regulation of CD1d represents the "physiologic inflammation" associated with a number of mucosal tissue sites (35). Some evidence exists that similar pathways may be associated with pathological processes. It was recently shown, for example, that increased expression of Hsp family members (especially Hsp70) in atherosclerotic lesions coincides with specific expression of CD1d, but not other MHC molecules (36). Whether such observations can be extended to other Hsp molecules is not known, but they suggest a relatively specific activation pathway for CD1d that involves Hsp-related molecules. Our studies also shed some light on this subject. Indeed, while IFN- $\gamma$ regulates both MHC class II and ICAM-1 on IECs (18), LC failed to modulate either of these molecules. Thus, additional work will be necessary to define the details of Hsp110-induced CD1d expression.

In summary, these studies identify a novel function elicited by LC of the intestinal milieu. Specifically, results from these studies suggest that epithelia may liberate Hsp110 and thus provide an autocrine signal for $\mathrm{CD} 1 \mathrm{~d}$ regulation on this cell type. These studies also predict a major role for Hsp110 in CD1d expression in other cells and provide evidence for yet another link between innate and adaptive immunity in the immunobiology of CD1d.

\section{Acknowledgments}

This work was supported by NIH grants DK-50189, DK-44319, DK-51362, and DK-53056 and the Harvard Digestive Diseases Center. R.S. Blumberg, L.F. Mayer, and R.B. Sartor were supported by a grant from the Broad Medical Research Program.

\footnotetext{
1. Blumberg, R.S., Gerdes, D., Chott, A., Porcelli, S.A., and Balk, S.P. 1995 Structure and function of the CD1 family of MHC-like cell surface proteins. Immunol. Rev. 147:5-29.

2. Briken, V., Moody, D.B., and Porcelli, S.A. 2000. Diversification of CD1 proteins: sampling the lipid content of different cellular compartments. Semin. Immunol. 12:517-525.

3. Exley, M., et al. 2000. CD1d structure and regulation on human thymocytes, peripheral blood $\mathrm{T}$ cells, B cells and monocytes. Immunology. 100:37-47.

4. Canchis, P.W., et al. 1993. Tissue distribution of the non-polymorphic major histocompatibility complex class I-like molecule, CD1d. Immunology. 80:561-565.

5. Blumberg, R.S., et al. 1991. Expression of a nonpolymorphic MHC class I-like molecule, CD1d, by a human intestinal epithelial cells. J. Immunol. 147:2518-2524

6. Saubermann, L.J., et al. 2000. Activation of natural killer T cells by alphagalactosylceramide in the presence of CD1d provides protection against colitis in mice. Gastroenterology. 119:119-128.
} 
7. Heller, F., Fuss, I.J., Nieuwenhuis, E.E., Blumberg, R.S., and Strober, W. 2002. Oxazolone colitis, a Th2 colitis model resembling ulcerative colitis, is mediated by IL-13-producing NK-T cells. Immunity. 17:629-638.

8. Colgan, S.P., Hershberg, R.M., Furuta, G.T., and Blumberg, R.S. 1999. Ligation of intestinal epithelial CD1d induces bioactive IL-10: critical role of the cytoplasmic tail in autocrine signaling. Proc. Natl. Acad. Sci. U. S. A. 96:13938-13943

9. Somnay-Wadgaonkar, K., et al. 1999. Immunolocalization of CD1d in human intestinal epithelial cells and identification of a beta2microglobulin-associated form. Int. Immunol. 11:383-392.

10. Colgan, S.P., Morales, V.M., Madara, J.L., Balk, S.P., and Blumberg, R.S 1996. IFN- $\gamma$ modulates CD1d expression on intestinal epithelia. Am.J. Physiol. 271:C276-C283.

11. Kim, H.S., et al. 1999. Biochemical characterization of CD1d expression in the absence of beta2- microglobulin. J. Biol. Chem. 274:9289-9295.

12. Exley, M., Porcelli, S., Furman, M., Garcia, J., and Balk, S. 1998. CD161 (NKR-P1A) costimulation of CD1d-dependent activation of human T cells expressing invariant $\mathrm{V}$ alpha $24 \mathrm{~J}$ alpha $\mathrm{Q} \mathrm{T}$ cell receptor alpha chains. J. Exp. Med. 188:867-876.

13. Wang, X.Y., et al. 2000. Characterization of native interaction of hsp110 with hsp25 and hsc70. FEBS Lett. 465:98-102.

14. Collard, C.D., et al. 2002. Neutrophil-derived glutamate regulates vascular endothelial barrier function. J. Biol. Chem. 2002:14801-14811.

15. Lee-Yoon, D., Easton, D., Murawski, M., Burd, R., and Subjeck, J.R. 1995. Identification of a major subfamily of large hsp70-like proteins through the cloning of the mammalian $110-\mathrm{kDa}$ heat shock protein. J. Biol. Chem. 270:15725-15733.

16. Parkos, C.A., et al. 1996. CD 47 mediates post-adhesive events required for neutrophil migration across polarized intestinal epithelia. J. Cell Biol. 132:437-450.

17. Schultz, M., et al. 1999. IL-2-deficient mice raised under germfree conditions develop delayed mild focal intestinal inflammation. Am. J. Physiol. 276:G1461-G1472.

18. Colgan, S.P., et al. 1994. Interferon- $\gamma$ induces a surface phenotype switch in intestinal epithelia: downregulation of ion transport and upregulation of immune accessory ligands. Am. J. Physiol. 267:C402-C410.

19. Parkos, C.A., et al. 1996. Expression and polarization of intercellular adhesion molecule- 1 on human intestinal epithelia: consequences for CD11b/18-mediated interactions with neutrophils. Mol. Med. 2:489-505.

20. Mizoguchi, E., Mizoguchi, A., and Bhan, A.K. 1997. Role of cytokines in the early stages of chronic colitis in TCR alpha-mutant mice. Lab. Invest. 76:385-397.

21. Panja, A., Blumberg, R.S., Balk, S.P., and Mayer, L. 1993. CD1d is involved in T cell-intestinal epithelial cell interactions. J. Exp. Med. 178:1115-1119.

22. Pitman, R.S., and Blumberg, R.S. 2000. First line of defense: the role of the intestinal epithelium as an active component of the mucosal immune system. J. Gastroenterol. 35:805-814.

23. Balk, S.P., et al. 1994. $\beta_{2}$-Microglobulin-independent MHC class Ib molecule expressed by human intestinal epithelium. Science. 265:259-262.

24. Canny, G., et al. 2002. Lipid mediator-induced expression of bacterici$\mathrm{dal} /$ permeability-increasing protein (BPI) in human mucosal epithelia. Proc. Natl. Acad. Sci. U. S. A. 99:3902-3907.

25. Li, Z., Menoret, A., and Srivastava, P. 2002. Roles of heat-shock proteins in antigen presentation and cross-presentation. Curr. Opin. Immunol. 14:45-51.

26. Ohashi, K., Burkart, V., Flohe, S., and Kolb, H. 2000. Cutting edge: heat shock protein 60 is a putative endogenous ligand of the toll-like receptor-4 complex. J. Immunol. 164:558-561.

27. Asea, A., et al. 2002. Novel signal transduction pathway utilized by extracellular HSP70: role of toll-like receptor (TLR) 2 and TLR4. J. Biol. Chem. 277:15028-15034.

28. Asea, A., et al. 2000. HSP70 stimulates cytokine production through a CD14-dependent pathway, demonstrating its dual role as a chaperone and cytokine. Nat. Med. 6:435-442.

29. Cario, E., et al. 2000. Lipopolysaccharide activates distinct signaling pathways in intestinal epithelial cell lines expressing Toll-like receptors. J. Immunol. 164:966-972.

30. Gewirtz, A.T., Navas, T.A., Lyons, S., Godowski, P.J., and Madara, J.L. 2001. Cutting edge: bacterial flagellin activates basolaterally expressed tlr5 to induce epithelial proinflammatory gene expression. J. Immunol. 167:1882-1885.

31. Pugin, J., et al. 1993. Lipolysaccharide activation of human endothelial and epithelial cells is mediated by lipopolysacchride-binding protein and soluble CD14. Proc. Natl. Acad. Sci. U. S. A. 90:2744-2748.

32. Becker, T., Hartl, F.U., and Wieland, F. 2002. CD40, an extracellular receptor for binding and uptake of Hsp70-peptide complexes. J. Cell Biol. 158:1277-1285.

33. Binder, R.J., Han, D.K., and Srivastava, P.K. 2000. CD91: a receptor for heat shock protein gp96. Nat. Immunol. 1:151-155.

34. Basu, S., Binder, R.J., Ramalingam, T., and Srivastava, P.K. 2001. CD91 is a common receptor for heat shock proteins gp96, hsp90, hsp70, and calreticulin. Immunity. 14:303-313.

35. Nagler-Anderson, C. 2001. Man the barrier! Strategic defences in the intestinal mucosa. Nat. Rev. Immunol. 1:59-67.

36. Bobryshev, Y.V., and Lord, R.S. 2002. Expression of heat shock protein70 by dendritic cells in the arterial intima and its potential significance in atherogenesis. J. Vasc. Surg. 35:368-375. 